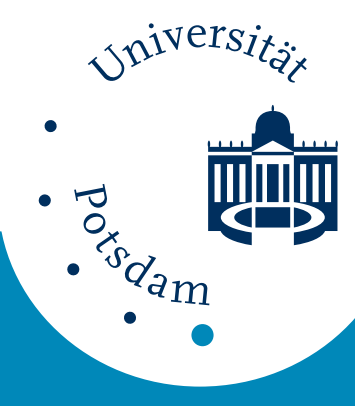

Universität Potsdam

Peter Imkeller, Sylvie Rœlly

\title{
Die Wiederentdeckung eines Mathematikers: Wolfgang Döblin
}

first published in:

Mitteilungen der Deutschen Mathematikervereinigung - 15 (2007),

p. $154-159$

ISSN: 0947-4471

Postprint published at the institutional repository of Potsdam University:

In: Postprints der Universität Potsdam :

Mathematisch-Naturwissenschaftliche Reihe ; 34

http://opus.kobv.de/ubp/volltexte/2007/1639/

http://nbn-resolving.de/urn:nbn:de:kobv:517-opus-16397

Postprints der Universität Potsdam

Mathematisch-Naturwissenschaftliche Reihe ; 34 


\section{Die Wieder- \\ entdeckung eines \\ Mathematikers: \\ Wolfgang \\ Döblin}

Peter Imkeller und Sylvie Rœlly

\section{Der versiegelte Brief und sein Inhalt}

Considérons une particule mobile se mouvant aléatoirement sur la droite (ou sur un segment de droite). Supposons qu'il existe une probabilité $F(x, y ; s, t)$ bien définie pour que la particule se trouvant à l'instant $s$ dans la position $x$ se trouve à l'instant $t(>s)$ à gauche de $y$, probabilité indépendante du mouvement antérieur de la particule.

Mit diesen Worten beginnt eines der bemerkenswertesten mathematischen Manuskripte des letzten Jahrhunderts. Es stammt vom Soldaten Wolfgang Döblin, Sohn des deutschen Schriftstellers Alfred Döblin, und trägt den Titel Sur l'équation de Kolmogoroff. Er verfasste es im Winter 1939/40 an der Front in Ostfrankreich. Seine Veröffentlichung verbindet sich mit einer unglaublichen Geschichte, die uns sprachlos machte, als wir zuerst davon hörten. Wolfgang Döblin, stationiert mit seiner Einheit in den Ardennen, später in den Vogesen, arbeitete während der Phase nach dem Überfall auf Polen, in Frankreich als la drôle de guerre bekannt, an diesem Manuskript. Er entschloss sich, es als versiegeltes Manuskript (pli cacheté) an das Archiv der Académie des Sciences in Paris zu schicken. Es war nicht das erste, das er auf diese Weise deponierte. Offenbar hatte er die Absicht, nach der Rückkehr aus einem von ihm als kurz eingeschätzten Krieg, die Arbeit daran fortsetzen und es zu publizieren. Ob er sich der Bedeutung seiner Erkenntnisse bewusst war, oder nur eifersüchtig versuchte, sie potentiellen Konkurrenten vorzuenthalten, bleibt Spekulationen überlassen. Döblin kehrte nie aus diesem Krieg zurück. Seine Einheit in

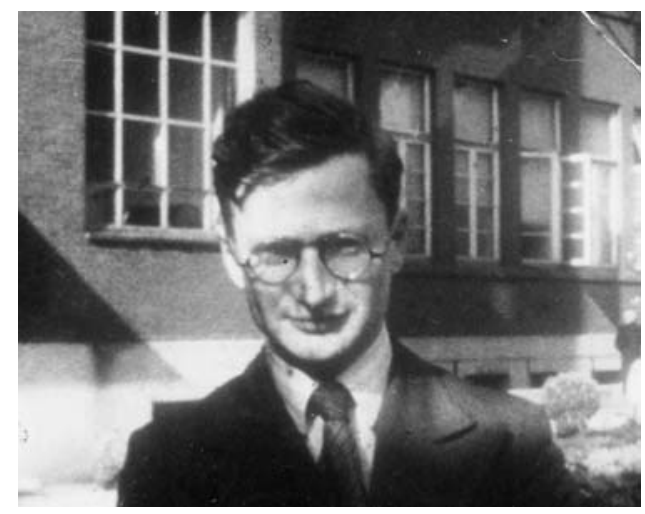

Wolfgang Döblin

der Auflösung, nahm er sich am 21. Juni 1940 in einer Scheune in Housseras in den Vogesen das Leben. Sein Manuskript blieb 60 Jahre unter Verschluss im Archiv und wurde erst im Jahre 2000 nach Zustimmung seines Bruders Stephan geöffnet. Wie weit Döblin damit seiner Zeit voraus war, wurde erkannt, nachdem es von Bernard Bru und Marc Yor ausgewertet worden war (CRAS, Série I, Tome 22I. N spécial Sur L'équation de Kolmogoroff, par W. Döblin. Décembre 2000).

Die Kolmogorov-Gleichung und ihre Interpretation

Der erste Satz umschreibt gleichzeitig das Programm des Manuskripts: „Wir betrachten ein bewegliches Teilchen, das sich zufällig auf der Geraden (oder einem Teil davon) bewegt. “ Döblin widmet sich damit der Aufgabe, die Fundamente eines Gebiets zu legen, das wir heute als stochastische Analysis bezeichnen. Er möchte ei- 
ne Lösung der eindimensionalen KolmogorovGleichung

$$
\begin{aligned}
&-v_{t}(x, t)=a v_{x}(x, t)+\frac{1}{2} \sigma^{2} v_{x x}(x, t), \\
& x \in \mathbb{R}, t \geq 0,
\end{aligned}
$$

$\left(v_{t}, v_{x}\right.$ bezeichnen im Folgenden Zeit- bzw. Ortsableitung einer Funktion $v(t, x)$ etc.) konstruieren, indem er die Beschreibung ihrer Dynamik aus den Begriffen der Kontinuumsmechanik löst, und in die der Stochastik übersetzt. Diese beschäftigt sich im Gegensatz zur Kontinuumsmechanik mit Bahnen idealisierter, zufällig bewegter diffundierender Teilchen. Die Realisierung dieses Vorhabens beginnt Döblin mit dem zweiten Satz: „Stellen wir uns eine wohldefinierte Wahrscheinlichkeit $F(x, y ; s, t)$ vor, mit der sich das Teilchen, das zu einem Zeitpunkt $s$ im Zustand $x$ ist, sich zum Zeitpunkt $t(>s)$ links des Zustands $y$ befindet, eine von der Vorgeschichte der Bewegung unabhängige Wahrscheinlichkeit.“ Döblin startet mit dem Begriff, den man aus heutiger Sicht als Verteilungsfunktion einer Übergangsdichte $p(x, y ; s, t)$ interpretieren könnte, verknüpft durch die Beziehung

$$
F(x, y ; s, t)=\int_{-\infty}^{y} p(x, z ; s, t) d z .
$$

Die Unabhängigkeit von der Vorgeschichte findet ihren Ausdruck in der Chapman-KolmogorovBeziehung, einer rudimentären Form der Markov-Eigenschaft. Sie besagt, dass für das zukünftige Verhalten des Teilchens, aus der Gegenwart gesehen, nur sein augenblicklich angenommener Zustand, nicht aber die Vorgeschichte seiner Bewegung, ausschlaggebend sind. Formal beschreibt sich diese Beziehung für Zustände $x, y$ und Zeitpunkte $0 \leq s \leq u \leq$ $t$ durch die Gleichung

$$
\begin{aligned}
& p(x, y ; s, t) \\
& =\int_{-\infty}^{\infty} p(z, y ; u, t) p(x, z ; s, u) d z .
\end{aligned}
$$

Übersetzt man sie in die Sprache der Kontinuumsmechanik, so gewinnt man eine Interpretation der Koeffizienten $a$ und $\sigma$ der Kolmogorov-Gleichung. Die Zusammenhänge sind die folgenden. Nach Definition von $F$ sollte für Zustände $x$ und Zeitpunkte $s$

$$
\begin{aligned}
a(x, s)=\lim _{t \downarrow s} & \frac{1}{t-s} \\
& \int_{|y-x|<1}(y-x) d F(x, y ; s, t)
\end{aligned}
$$

den infinitesimalen erwarteten Trend der Bewegung, d.h. die Drift des diffundierenden Teilchens, und

$$
\begin{aligned}
\sigma^{2}(x, s)= & \lim _{t \downarrow s} \frac{1}{t-s} \\
& \int_{|y-x|<1}(y-x)^{2} d F(x, y ; s, t)
\end{aligned}
$$

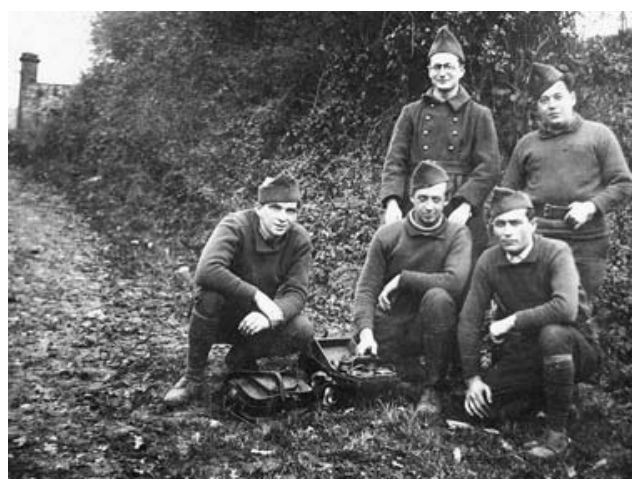

Wolfgang Döblin hinten links

die infinitesimale Varianz der Bewegung, ihre Diffusionsintensität oder in heutiger Sprechweise ihre Volatilität wiedergeben.

Eine trajektorielle Lösung der Kolmogorov-Gleichung

Ausgehend von dieser Interpretation stellt Döblin als erster eine trajektorielle Lösung der Kolmogorov-Gleichung auf, eine Zustandsgleichung, die man in eine stochastische Integralgleichung im Sinne des Itô'schen Kalküls übersetzen kann. Ist $X_{t}$ die zufällige Position des idealisierten Teilchens zur Zeit $t$, so gilt nach Döblin

$$
X_{t}=x+\beta_{H_{t}}+\int_{0}^{t} a\left(s, X_{s}\right) d s,
$$

wobei $\beta_{H_{t}}$ eine in der Zeitskala $H_{t}=$ $\int_{0}^{t} \sigma^{2}\left(s, X_{s}\right) d s$ laufende Brownsche Bewegung $\beta$ ist. Erst nach Konstruktion des stochastischen Integrals der Brownschen Bewegung findet K. Itô etwa fünf Jahre später zu seiner Beschreibung dieser ersten trajektoriellen Lösung der Kolmogorov-Gleichung in der Form

$$
\begin{aligned}
X_{t}=x+\int_{0}^{t} \sigma\left(s, X_{s}\right) & d B_{s} \\
& +\int_{0}^{t} a\left(s, X_{s}\right) d s .
\end{aligned}
$$

Itô erhielt beim ICM in Madrid im August 2006 den ersten Gauss-Preis für seine bahnbrechenden Arbeiten in der stochastischen Analysis. Die Übersetzung zwischen (I) und (2) liefert aus heutiger Sicht ein erst 1969 von Dubins und Schwarz bewiesener Satz von der Zeittransformation der Brownschen Bewegung. In einer allgemeineren Form besagt er, dass ein stetiges lokales Martingal $M$ mit quadratischer Variation $\langle M\rangle$ aufgefasst werden kann als $\beta_{\langle M\rangle}$, d. h. eine Brownsche Bewegung $\beta$, die in der für $M$ intrinsischen Zeitskala $\langle M\rangle$ abläuft. Wendet man diesen Satz auf das stetige lokale Martingal $\int_{0} \sigma\left(s, X_{s}\right) d B_{s}$ mit quadratischer Variation $\int_{0} \sigma^{2}\left(s, X_{s}\right) d s$ an, so kommt man von der 
Itô'schen Version der Lösung der Kolmogorov'schen Gleichung gerade zu der von Döblin. Döblin hat also zu seiner Beschreibung der trajektoriellen Lösung der Kolmogorov-Gleichung die Intervention des Itô'schen Integrals dadurch umgangen, dass er implizit einen erst viel später geklärten Zusammenhang zwischen Martingalen und zeittransformierten Brownschen Bewegungen vorwegnahm.

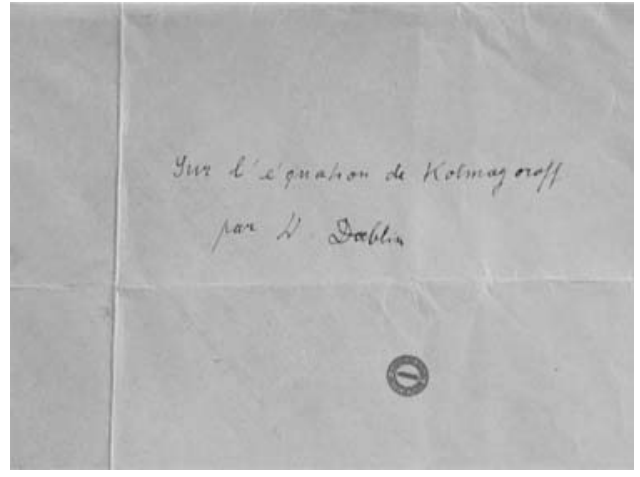

Der Hauptsatz der stochastischen Analysis

Die aus heutiger Sicht interessanteste und spektakulärste Erkenntnis gewinnt Döblin in Abschnitt XV seines Manuskripts. Dort findet er eine Version des heute als Itô-Formel bekannten Hauptsatzes der stochastischen Analysis, und zwar wieder, indem er das stochastische Integral der Itô'schen Version durch eine zeittransformierte Brownsche Bewegung mit der richtigen Geschwindigkeit ersetzt. In der Itô'schen Version erhält man für eine Funktion $f$, die einmal stetig in der Zeitvariablen, zweimal stetig in der Raumvariablen differenzierbar ist, und eine trajektorielle Lösung $X$ der Kolmogorov-Gleichung (2) die Formel

$$
\begin{array}{r}
f\left(t, X_{t}\right)=f(0, x)+\int_{0}^{t}\left[f_{x} \sigma\right]\left(s, X_{s}\right) d B_{s} \\
+\int_{0}^{t}\left[f_{x} a+f_{t}+\frac{1}{2} f_{x x} \sigma^{2}\right]\left(s, X_{s}\right) d s .
\end{array}
$$

Döblin kommt in seinem Manuskript etwa fünf Jahre vor Itô auf die alternative, das stochastische Integral vermeidende Beschreibung

$$
\begin{aligned}
f\left(t, X_{t}\right) & =f(0, x)+\beta_{H_{t}^{t}} \\
& +\int_{0}^{t}\left[f_{x} a+f_{t}+\frac{1}{2} f_{x x} \sigma^{2}\right]\left(s, X_{s}\right) d s,
\end{aligned}
$$

wobei die Brownsche Bewegung $\beta$ mit der Geschwindigkeit

$$
H_{t}^{f}=\int_{0}^{t}\left[f_{x} \sigma\right]^{2}\left(s, X_{s}\right) d s
$$

läuft.
Von der Leibniz-Newton-Formel zur Döblin-Itô-Formel

Um die Bedeutung dieser Formeln für die Mathematik zu ermessen, betrachten wir den einfachen Spezialfall der elementarsten Kolmogorov-Gleichung

$$
-v_{t}=\frac{1}{2} v_{x x}
$$

d.h. der klassischen Wärmeleitungsgleichung. Ihre trajektorielle Beschreibung führt mit der Wahl $a=0, \sigma=1$ auf die Brownsche Bewegung selbst, d. h. $X=B$. Erinnern wir zunächst an den Hauptsatz der klassischen Infinitesimalrechnung, d. h. die Formel, die man Leibniz und Newton zuschreiben kann. Ist $x:[0, t] \rightarrow \mathbb{R}$ eine stetig differenzierbare Trajektorie (der klassischen Analysis), $f: \mathbb{R} \rightarrow \mathbb{R}$ eine stetig differenzierbare Funktion, so besagt dieser Hauptsatz

$$
\begin{aligned}
f\left(x_{t}\right)-f\left(x_{0}\right) & =\int_{0}^{t} f^{\prime}\left(x_{s}\right) v_{s} d s \\
& =\int_{0}^{t} f^{\prime}\left(x_{s}\right) d x_{s}
\end{aligned}
$$

wenn $v$ die Geschwindigkeit des Teilchens mit Trajektorie $x$ ist, d.h. $v=x^{\prime}$ gilt. Hierbei taucht als $d x$ das intrinsische zu $x$ gehörige Längenmaß der Kurve $x$ auf. Man kann die Leibniz-Newton-Formel beweisen, indem man ihre Aussage entlang einer Folge von Partitionen von $[0, t], z$. B. durch äquidistante Punkte $t_{i}=\frac{i}{n} t, 0 \leq i \leq n, n \in \mathbb{N}$, in einer Taylorentwicklung approximiert. Diese Approximation führt zu

$$
\begin{aligned}
& f\left(x_{t}\right)-f\left(x_{0}\right) \\
& =\sum_{i=0}^{n-1}\left[f \left(x_{t_{i+1}}-f\left(x_{t_{i}}\right]\right.\right. \\
& =\sum_{i=0}^{n-1} f^{\prime}\left(x_{t_{i}}\right)\left(x_{t_{i+1}}-x_{t_{i}}\right)+R_{n}
\end{aligned}
$$

mit einem asymptotisch verschwindenden Restterm $R_{n}$.

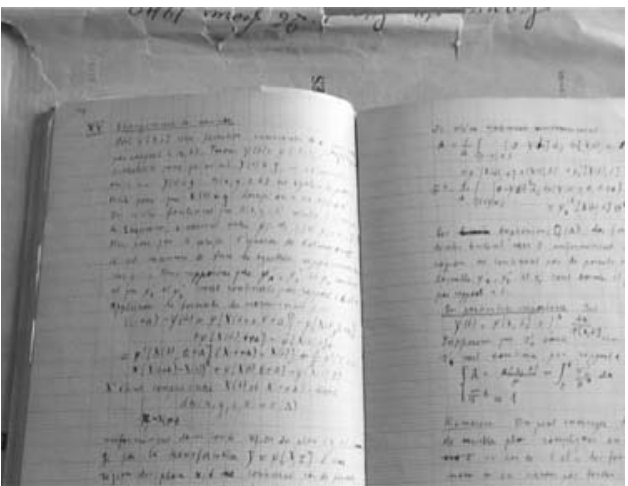


Für die Trajektorien von Teilchen der klassischen Mechanik, kann man eine Geschwindigkeit als Ableitung der Bewegungskurve festlegen. Insbesondere haben solche Trajektorien beschränkte Variation, die gegeben ist durch

$$
\begin{aligned}
|x|_{t} & =\lim _{n \rightarrow \infty} \sum_{i=0}^{n-1}\left|x_{t_{i+1}}-x_{t_{i}}\right| \\
& =\int_{0}^{t}\left|v_{s}\right| d s .
\end{aligned}
$$

Die typischen Trajektorien einer Lösung der Kolmogorov'schen Gleichung oder der Brownschen Bewegung sind viel rauher als die Teilchenbahnen der klassischen Mechanik. Sie besitzen weder Geschwindigkeit noch beschränkte Variation, sind in der Regel nur Hölder-stetig von Ordnung $\frac{1}{2}-\varepsilon$ für $\varepsilon>0$, was sich auch darin ausdrückt, dass nur ihre quadratische $\mathrm{Va}$ riation oder Variation zweiter Ordnung endlich ist, und gegeben wird durch

$$
\lim _{n \rightarrow \infty} \sum_{i=0}^{n-1}\left(B_{t_{i+1}}-B_{t_{i}}\right)^{2}=t .
$$

Daher muss man in einer analogen Approximation einer die Differential- und Integralrechnung verbindenden Fundamentalformel der stochastischen Analysis einen Schritt weiter gehen. Ist wieder eine glatte Funktion $f$ gegeben, mit der man die Pfade transformiert, d.h. die Bilder $f\left(B_{t}\right), t \geq 0$, betrachtet, und approximiert man wie oben die Differenz von $f\left(B_{t}\right)-$ $f\left(B_{0}\right)$ durch die Taylor-Formel entlang der äquidistanten Partitionenfolge, so muss man in der pfadweisen Entwicklung Terme bis zur Differentiationsordnung 2 berücksichtigen:

$$
\begin{aligned}
& f\left(B_{t}\right)-f\left(B_{0}\right)=\sum_{i=0}^{n-1}\left[f \left(B_{t_{i+1}}-f\left(B_{t_{i}}\right]\right.\right. \\
& =\sum_{i=0}^{n-1} f^{\prime}\left(B_{t_{i}}\right)\left(B_{t_{i+1}}-B_{t_{i}}\right) \\
& \quad+\sum_{i=0}^{n-1} \frac{1}{2} f^{\prime \prime}\left(B_{t_{i}}\right)\left(B_{t_{i+1}}-B_{t_{i}}\right)^{2}+R_{n} .
\end{aligned}
$$

Man zeigt dann, dass $\operatorname{der} f^{\prime}$ enthaltende Term gegen das Itô'sche Integral $\int_{0}^{t} f^{\prime}\left(B_{s}\right) d B_{s}$, der zweite Summenterm gegen das Integral von $f^{\prime \prime}(B)$ bezüglich der quadratischen Variation der Brownschen Bewegung, das heißt $\frac{1}{2} \int_{0}^{t} f^{\prime \prime}\left(B_{s}\right) d s$ konvergiert. Damit ergibt sich die Itô'sche Formel für die Brownsche Bewegung

$$
\begin{aligned}
f\left(B_{t}\right) & -f\left(B_{0}\right) \\
= & \int_{0}^{t} f^{\prime}\left(B_{s}\right) d B_{s}+\frac{1}{2} \int_{0}^{t} f^{\prime \prime}\left(B_{s}\right) d s
\end{aligned}
$$



die in der Döblin'schen Version die Form

$$
\begin{aligned}
f\left(B_{t}\right)-f\left(B_{0}\right) & \\
& =\beta_{H_{t}^{t}}+\frac{1}{2} \int_{0}^{t} f^{\prime \prime}\left(B_{s}\right) d s,
\end{aligned}
$$

mit einer mit der Geschwindigkeit

$$
H_{t}^{f}=\int_{0}^{t}\left(f^{\prime}\right)^{2}\left(B_{s}\right) d s
$$

laufenden Brownschen Bewegung $\beta$ annimmt. Die Döblin-ltô-Formel kann also als das zentrale Ergebnis der stochastischen Analysis aufgefasst werden, das den Hauptsatz der Infinitesimalrechnung nach Leibniz und Newton in den Bereich der Trajektorien stochastischer Prozesse verallgemeinert. Ohne sie wäre der elegante Martingalansatz zur stochastischen Lösung des Merton-Scholes-Problems der Finanzmathematik undenkbar.

\section{Ein Meilenstein}

Wie weit Döblin seiner Zeit voraus war, kann man auch noch an anderen Beobachtungen aus dem Manuskript erkennen. So diskutiert er in einem der letzten Abschnitte eine Version eines Vergleichssatzes, mit der Aussage, dass die Trajektorien zweier Lösungen der Kolmogorov-Gleichung mit Driftkoeffizienten $a_{1} \leq a_{2}$ die durch die Driftkoeffizienten vorgegebene Ordnung beibehalten. Dieser Satz wird in der Literatur der stochastischen Analysis Arbeiten von Yamada (1973) und Yamada-Ogura (I98I) zugeschrieben. Das pli cacheté ist ein Meilenstein der stochastischen Analysis, der für die Entwicklungsgeschichte des Gebiets groteskerweise ohne Bedeutung blieb, da er 60 Jahre lang verborgen war.

\section{Die Veröffentlichung der Geschichte}

Die mit seiner Entstehung verbundene unglaubliche Geschichte hat nicht nur Mathematiker bewegt und berührt, sondern fand bald nach 
der Entdeckung viel Resonanz in einer breiteren Öffentlichkeit. Zuerst in Frankreich, mit einer zeitlichen Verzögerung auch in Deutschland, wurde sie von Journalisten vor allem in Wissenschaftsredaktionen in der Presse, im Rundfunk und Fernsehen aufgegriffen. Der Dokumentarfilm von Jürgen Ellinghaus und Hubert Ferry Der versiegelte Brief des Soldaten Döblin, eine Koproduktion von ARTE und dem RBB, wurde vor einigen Monaten in ARTE, und erst vor kurzem bei RBB zum ersten Mal ausgestrahlt. Grosse Begeisterung für die Geschichte und das Schicksal von Wolfgang Döblin begleitete auch die Recherchen von Agnes Handwerk und Harrie Willems, die sich in einem Film niederschlagen, den wir hier kurz vorstellen möchten.

\section{Besprechung des Films Wolfgang Döblin - ein Mathematiker wird wiederentdeckt I'}

Der Film von Agnes Handwerk und Harrie Willems illustriert beispielhaft, unter welchen auBergewöhnlichen Bedingungen Wolfgang Döblin - zweitältester Sohn des Schriftstellers sein geniales mathematisches Werk geschaffen hat. Dabei beschreibt er aber auch viel mehr: Im Hintergrund handelt es sich um die Beziehung zwischen Vater und Sohn, Literatur und Mathematik, rassistischer Politik und Wissenschaft, Krieg und Tod. Dieser Film führt das tragische Schicksal eines herausragenden Wissenschaftlers vor Augen, dessen Genie erst 60 Jahre nach seinem Tod dank der Öffnung eines versiegelten Briefes allgemein bekannt wurde. Wir hatten das Glück, vor kurzem den Journalisten und Filmemachern zu begegnen und sie über die Entstehung des Films zu befragen. Unsere Diskussion werden wir skizzieren, ohne alle Details der Geschichte Wolfgang Döblins zu verraten!

Wie Agnes Handwerk und Harrie Willems auf die Idee gekommen sind, einen Film über W. Döblin zu drehen...

In der lokalen Presse lesen die beiden Journalisten 2000 in einem Artikel folgendes: Die französischen Wahrscheinlichkeitstheoretiker Bernard Bru und Marc Yor hätten vor kurzem einen seit sechzig Jahren verschlossenen Brief mit der Aufschrift Sur l'équation de Kolmogoroff von Wolfgang Döblin geöffnet; sie hätten das Manuskript entschlüsselt und dabei festgestellt, dass der Autor 1939 eine bahnbrechende Theorie der Differential- und Integralrechnung von zufälligen Prozessen initiiert habe, die dann Jahre später vom japanischen Mathematiker Kiyoshi Itô unabhängig entwickelt worden sei. Eine Sensation für die Welt der Mathematiker! Sofort stellen sich ihnen Fragen. Unter anderen: Warum die Öffnung erst jetzt? Was für ein Mensch war Wolfgang Döblin? Warum sind seine mathematischen Ergebnisse wichtig?

\section{Die Genesis des Films}

Spontan interessiert an dieser außergewöhnlichen Geschichte fangen die beiden Journalisten an, Informationen zu sammeln. Das erste entscheidende Treffen findet mit Bernard Bru, Professor an der Universität Paris V, statt. Er erzählt, wie er bei der Vorbeitung für eine Konferenz in den USA zum Gedenken an Wolfgang Döblin - organisiert von den berühmten Mathematikern Kai Lai Chung und Joe Doob 1991 in Paris an der Académie des Sciences auf die Existenz eines versiegelten Briefes gestoßen sei, und wie kompliziert der Weg bis zur Öffnung dieses Briefes gewesen sei. Er betont, wie aktuell und wichtig diese mathematische Arbeit ist: Es handelt sich um die Analyse von Transformationen der Brownschen Bewegung, die heutzutage zum Beispiel in der Finanzmathematik eine zentrale Rolle spielt. Bernard Bru ermutigt Agnes Handwerk und Harrie Willems sehr, darüber eine dokumentarische Arbeit zu verfertigen, und stellt großzügigerweise viel Material zu Verfügung. Es entsteht zunächst ein Beitrag für den Hörfunk und dann ein Videofilm. Eine DVD mit den zwei Teilen des Films ist vor kurzem im Springer-Verlag erschienen. Da weder eine deutsche noch eine französische Institution oder Stiftung sich bereit erklärte, das Projekt auch nur teilweise zu finanzieren, müssen die Journalisten mit einfachen Mitteln arbeiten.

\section{Konzeption des Films}

Es gelingt Agnes Handwerk und Harrie Willems, mehrere Zeitzeugen und Wolfgangs Brüder zu interviewen. So lernt man im Film Claude und Stephan Doblin ${ }^{2}$ kennen, genau so wie Frau Piron-Lévy, Tochter des Mathematikers Paul Lévy. All diese Begegnungen sind für den Zuschauer bewegend, insbesondere, weil diese älteren Menschen noch sehr präzise über Erlebnisse sprechen können, die sich schon vor dem zweiten Weltkrieg zugetragen haben. Wichtig ist es für die Regisseure, uns Wolfgang

I Im Teil II des Films erklärt der Professor Marc Yor aus der Universität Paris VI den mathematischen Inhalt des Manuskripts von W. Döblin „Über die Kolmogoroff-Gleichung“ und diskutiert die Übereinstimmungen mit dem stochastischen Kalkül von K. Itô.

2 Nach ihrer Einbürgerung in Frankreich haben Wolfgang, Klaus und Stephan Döblin ihre Namen in Vincent Doeblin, Claude und Stephan Doblin geändert. 
Döblin nicht nur als Wissenschaftler, sondern auch als Menschen zu präsentieren. Der familiäre und intellektuelle Kreis, in dem er in Berlin und in Paris lebte, war sehr lebendig, und das Engagement des jungen Wolfgang kompromisslos. Man versteht im Laufe des Films, wie ihm seine mathematische Aktivität im Krieg bis zu einem bestimmten Moment geholfen hat zu überleben. Als einfacher Telegrafist an der französischen Front 1939-40 fühlte er sich extrem isoliert. Trotz der widrigen Umgebung hat er sich jedoch nie entmutigen lassen. Die spannenden Probleme der Mathematik haben nie aufgehört, ihn zu beschäftigen. Damit konnte er der grausamen Realität entfliehen, getragen von der Schönheit der abstrakten ChapmanKolmogorov Gleichung.

\section{Realisierung des Films}

Agnes Handwerk und Harrie Willems wurde während der Dreharbeit des Films von vielen Seiten Bereitschaft und Kooperation entgegen gebracht: Claude und Stephan Doblin ließen sich lange interviewen; die Einwohner des Dorfes Housseras in den Vogesen, wo das Leben von Wolfgang im Juni 1940 ein Ende fand, waren offen und gesprächsbereit. Auch der Bürgermeister freute sich über das Projekt, stolz, dass Mitglieder der berühmten Familie Döblin in seinem Friedhof ruhen, und dass der Name Vincent Doblin auf dem Denkmal für die Gefallenen des Dorfes steht. Man hätte fast vergessen können, dass die Döblins Deutsche waren, und dass deswegen in früheren Zeiten der Familie gegenüber eine ganz andere Stimmung herrschte...

Die schwierige Nachkriegszeit und ihre Folgen für die Spuren, die Wolfgang hinterliess

Als die Döblins 1933 in Eile und zwangsweise von Berlin nach Paris emigrierten, war es für jeden aus der Familie schwierig, sich im fremden Land einzuleben. Besonders traf das auf Alfred zu, der die französische Sprache schlecht beherrschte. Immerhin erhielten sie Hilfe von einflussreichen Personen. Wolfgang traf in Paris an der Sorbonne auf eine großen Gruppe von Mathematikern - unter anderen Fréchet, Lévy, Darmois, Hadamard, Loève - die seine große Begabung schätzten. Er wurde respektiert und integriert. (Er behielt im Französischen immer einen sehr starken Akzent. In der französischen Armee erklärte er ihn gegenüber seinen $\mathrm{Ka}$ meraden damit, dass er aus dem Elsass käme!) Nach dem Krieg aber war die französische Gesellschaft Deutschen gegenüber sehr misstrauisch, auch gegenüber Emigranten, die direkt un- ter den Nazis gelitten hatten wie die Döblins. Die Brüder von Wolfgang haben diese bittere Erfahrung gemacht, auch die Mutter Erna Döblin, als sie jährlich zum Friedhof in Housseras fuhr, und dort feindselig angesehen wurde. Und das Werk von Wolfgang? Vermutlich liegt ein Grund für das Vergessen des versiegelten Briefes in dieser negativen Stimmung der Nachkriegszeit allen Deutschen gegenüber. In Frankreich vergingen Jahrzehnte, um die Kriegszeit objektiver zu sehen und das Leid vieler Deutscher anzuerkennen.

\section{Abschließende Bemerkung}

Der versiegelte Brief von Wolfgang Döblin mit seinen grundlegenden mathematischen Neuerungen brauchte fünfzig Jahre, um wiederentdeckt zu werden, und fast ein weiteres Jahrzehnt, um geöffnet zu werden. Die Dreharbeiten für den Film benötigten einige weitere Jahre. Dass Döblins Arbeit heute der französischen und deutschen Öffentlichkeit vorliegt und so viel Interesse weckt, ist ein ermutigendes Zeichnen der Versöhnung zwischen Frankreich und Deutschland. Wolfgang Döblin hat beide Länder geliebt und aus den mathematischen Kulturen beider Länder die ihm eigene Summe gebildet.

\section{Der Film}

„Wolfgang Doeblin - ein Mathematiker wird wiederentdeckt"

DVD, Springer-Verlag Berlin

Teil I Biographie 55 Min.

Teil 2 „Sur l'équation de Kolmogorov“ 25 Min. ISBN: 978-3-540-7I959-5.

\section{Bildnachweise}

Deutsches Literaturarchiv, Marbach. Der Abdruck der Photographien erfolgt mit freundlicher Genehmigung von Stephan Doblin.

\section{Adresse der Autoren}

Prof. Dr. Peter Imkeller

Institut für Mathematik

Humboldt Universität zu Berlin

Unter den Linden 6

10099 Berlin

imkeller@mathematik.hu-berlin.de

Prof. Dr. Sylvie Rœlly

Institut für Mathematik

Universität Potsdam

Am neuen Palais 10

I4469 Potsdam

roelly@math.uni-potsdam.de 